'Departamento de Dermatología, Escuela de Medicina, Pontificia Universidad Católica de Chile. Santiago, Chile.

${ }^{2}$ Escuela de Medicina, Universidad de Santiago de Chile. Santiago, Chile.

${ }^{3}$ Sección de Medicina de Urgencia, Departamento de Medicina Interna, Escuela de Medicina, Pontificia Universidad Católica de Chile. Santiago, Chile.

aMagíster en Epidemiología,

Trabajo no recibió financiamiento. Los autores declaran no tener conflictos de interés.

Recibido el 29 de septiembre de 2020, aceptado el 16 de marzo de 2021.

Correspondencia a: David Acuña Ramírez dgacuna@uc.cl

\section{Caracterización clínica y epidemiológica de 200 pacientes con loxoscelismo cutáneo y cutáneo visceral}

\author{
KATHERINE DROPPELMANN ${ }^{1}$, PAULA MAJLUF-CÁCERES ${ }^{1}$, \\ NATALIA SABATINI-UGARTE ${ }^{1}$, ELIZABETH VALLE ${ }^{1}$, \\ HERNÁN HERRERA ${ }^{2, a}$, DAVID ACUÑA ${ }^{3}$
}

\section{Loxoscelism. Experience in 200 patients}

Background: Loxoscelism is an important public health problem in Chile and South America, due to the higher rate of cutaneous-visceral involvement. The diagnosis of loxoscelism is mostly clinical without established diagnostic criteria. There is little evidence to support any treatment used in this condition. Aim: To characterize the clinical features and epidemiology of loxoscelism among patients consulting at the Emergency and Dermatology Services of a clinical hospital between 2013 and 2017. Material and Methods: Review of medical records of patients registered in the electronic clinical record system with a confirmed diagnosis of loxoscelism. Epidemiological, clinical, laboratory tests and treatment variables were analyzed. Results: We reviewed data from 200 patients. Ninety-four percent presented cutaneous loxoscelism and 5.5\% cutaneous-visceral loxoscelism. Systemic symptoms were present in $73 \%$ of patients with cutaneous-visceral loxoscelism. Patients who developed systemic symptoms had an 18 times higher risk of developing cutaneous-visceral loxoscelism. Laboratory abnormalities were more common in patients with cutaneous-visceral loxoscelism. Not all patients with hematuria had cutaneous-visceral loxoscelism. Most patients required analgesia. Anti-loxosceles serum was not used in any patient. Conclusions: Many questions remain to be answered regarding the diagnosis and treatment of the disease. Studies are required to validate diagnostic criteria for loxoscelism, predictors for visceral involvement and response to treatment.

(Rev Med Chile 2021; 149: 682-688)

Key words: Brown Recluse Spider; Spider Bites; Spider Venoms.
$\mathrm{E}$ xisten dos síndromes clínicos clásicos causados por mordeduras de araña: latrodectismo (causado por Latrodectus spp) y loxoscelismo (causado por Loxosceles spp) (1,2 $^{1,}$.

La distribución de las arañas del género Loxosceles es amplia. La Loxosceles reclusa es la causante de la mayoría de los casos en Norteamérica ${ }^{3-12}$. La Loxosceles laeta, intermedia y gaucho son causantes de la mayoría de los casos en Sudamérica ${ }^{13}$.

La presencia de L. laeta en nuestro país determina que el loxoscelismo sea un problema relevante de salud pública, por la mayor tasa de compromiso cutáneo-visceral asociado al veneno de esta araña (propiedades necrosantes, hemolíticas, vasculíticas y coagulantes) ${ }^{14-16}$. Las características de estas lesiones fueron descritas en 1934 en Chile por el Dr. Atilio Macchiavello, describiendo la mordedura de la L. laeta como la mancha gangrenosa de Chile, dando a conocer los tipos clínicos que dependen de la gravedad de la inoculación del veneno y publicando en 1947 una imagen de la lesión del veneno necrotizante de este arácnido, dando sus características y evolución en el tiempo ${ }^{17,18}$. 
En el año 2005, el Centro de Atención Toxicológica de la Pontificia Universidad Católica de Chile atendió 2.831 llamados telefónicos de pacientes con sospecha de loxoscelismo, confirmándose 287 de ellos ${ }^{19}$. En el Hospital Clínico de la Universidad de Chile se siguieron y confirmaron 250 casos hospitalizados entre los años 1955 y 2000, con 56 casos pediátricos ${ }^{20}$.

El diagnóstico de loxoscelismo normalmente es clínico. La presencia de una lesión cutánea e historia epidemiológica compatible, suelen considerarse suficientes para establecer un diagnóstico de probable. Se puede tener certeza del diagnóstico cuando la araña es identificada y examinada por un experto ${ }^{21,22}$.

Se han descrito dos cuadros clínicos asociados a la mordedura de esta araña. El loxoscelismo cutáneo (LC) (83,3\% del total de casos) y el loxoscelismo cutáneo-visceral (LCV) (13-16\% del total de casos) $)^{2}$.

El LC es un cuadro limitado, generalmente benigno, caracterizado por dolor, edema y eritema cutáneo (primeras 6 a 8 h de la mordedura), que puede tener una ampolla central, y progresa a un halo de vasoconstricción e isquemia que se extiende alrededor de la lesión. A los 5-7 días se forma la placa livedoide que evoluciona con ulceración y necrosis. El tejido necrótico se desprende después de 2-3 semanas, dejando una úlcera con tejido granulatorio que puede tardar semanas o meses en cicatrizar ${ }^{14,23}$. La forma cutánea se puede asociar a síntomas sistémicos inespecíficos (hasta $50 \%$ de los casos en las primeras 24-48 h). También se ha descrito una variante edematosa de $\mathrm{LC}^{1,24,25}$.

El LCV se caracteriza por compromiso sistémico, caracterizado por compromiso del estado general, cefalea, mialgias, náuseas, vómitos, ictericia y fiebre. Estos síntomas se presentan normalmente dentro de las primeras $24 \mathrm{~h}$. En los exámenes se evidencia hemólisis, hematuria, hemoglobinuria, ictericia, insuficiencia renal, anemia. El compromiso sistémico no se correlaciona con la gravedad de las manifestaciones cutáneas ${ }^{1,2,11,14,26}$.

No existen exámenes de laboratorio que confirmen el diagnóstico de LCV, pero lo más importante es determinar la presencia de hemólisis, hemoglobinuria o hematuria ${ }^{19}$.

Se han descrito diversos tratamientos para el loxoscelismo, pero hay poca evidencia para apoyar cualquiera de ellos, incluido el papel del suero antiloxosceles y el momento de su uso ${ }^{1,14}$.
A pesar de que el loxoscelismo es un problema importante de salud pública en Chile, y que genera gran impacto en la población, no existen muchos datos estadísticos que muestren la real incidencia del problema. Falta mucha información respecto a la epidemiología y tratamiento.

En este contexto se diseñó un estudio, en el que se analizaron los datos de las fichas clínicas de los pacientes con LC y LCV consultantes en los departamentos de Dermatología y en el Servicio de Urgencia de la Red de Salud UC-Christus entre los años 2013 y 1017.

El objetivo principal fue caracterizar la clínica y epidemiología del LC y LCV en pacientes atendidos durante este período.

Además, los datos obtenidos en este estudio podrían orientarnos a realizar futuros estudios prospectivos al respecto y ver qué variables se podrían estudiar para poder contribuir al tratamiento y diagnóstico de estos casos.

\section{Material y Método}

Este estudio fue aprobado por el Comité de Ética en Investigación de la Pontificia Universidad Católica de Chile.

Se realizó un estudio observacional retrospectivo, para lo cual se analizaron todas las consultas por sospecha de mordedura de araña de rincón (Loxosceles laeta) en el Servicio de Urgencias o en Dermatología de la Red de Salud UC-Christus, registrados en el sistema de ficha clínica electrónica "ALERT", entre enero del año 2013 y diciembre del 2017. Se seleccionaron a aquellos pacientes registrados con diagnóstico confirmado de LC (descripción clínica cutánea clásica o mordedura + examen morfológico del arácnido compatible). Se seleccionaron y consideraron como LCV a aquellos pacientes con clínica cutánea clásica o mordedura + examen morfológico del arácnido compatible + diagnóstico explícito en la ficha clínica de LCV + evolución compatible. En los pacientes seleccionados se analizaron las siguientes variables: sexo, edad, fecha de la mordedura, ubicación geográfica, horas de evolución antes de la consulta, zona de la mordedura, signos clínicos de presentación, infección secundaria, resultados de exámenes de laboratorio solicitados, tipo de loxoscelismo y tratamiento realizado. Además, se determinó la proporción de cuadros cutáneos clásicos, cutáneos edematosos y cutáneos-viscerales. 


\section{Análisis estadístico}

Se realizó un análisis descriptivo de todas las variables consideradas, expresando como proporciones las variables categóricas y como media las variables continuas. Para analizar la relación entre las variables categóricas se utilizó el estadístico chi cuadrado. Se consideró significativo todo valor $\mathrm{p}$ menor o igual a 0,05. Los análisis estadísticos fueron realizados en el programa estadístico STATA versión 14.

\section{Resultados}

Se revisaron un total de 348 casos entre enero de 2013 y diciembre de 2017. De los 348 casos, se incluyeron 200 casos según los criterios diagnósticos establecidos. Ciento cuarenta y ocho casos fueron excluidos porque no cumplían con los criterios diagnósticos de loxoscelismo o por información incompleta en la ficha clínica.

Las edades de los pacientes fluctuaron entre los 3 y 87 años, con una media de 40 años. El 55,5\% de los pacientes era de sexo femenino. La mayor proporción de individuos fueron adultos con LC clásico. El 81,5\% del total de pacientes refirió una moderdura o visualizó la araña. La mayoría de las mordeduras ocurrieron en los meses de noviembre, diciembre, enero y febrero. La localización más frecuente fue extremidades inferiores, seguida por extremidades superiores y abdomen (Tabla 1 ).

El 94,5\% de los pacientes presentó LC (89,5\% LC clásico y 5\% LC edematoso) y 5,5\% LCV (Tabla 1). No hubo muertes por loxoscelismo. Con respecto a los subtipos cutáneo y cutáneo visceral, no existieron diferencias estadísticamente significativas entre adultos $(94,7 \%$ y $5,4 \%)$ y niños $(92,3 \%$ y $7,7 \%)$, ni entre hombres (91\% y $9 \%)$ y mujeres $(97,3 \%$ y $2,7 \%)$ en la ocurrencia de este último ( $\mathrm{p}=0,72$ y $\mathrm{p}=0,053$, respectivamente). No hubo asociación significativa entre la localización de la mordedura y la presencia de compromiso visceral $(\mathrm{p}=0,12)$.

El 32\% de los pacientes con síntomas sistémicos evolucionó a un LCV y 1,7\% de los pacientes sin síntomas sistémicos evolucionó a un LCV ( $\mathrm{p}<0,001$ ). El 72,7\% de los pacientes con LCV presentó síntomas sistémicos $(\mathrm{p}<0,001)$. De ellos, los más frecuentes fueron fiebre y síntomas gastrointestinales (Tabla 2). Los pacientes que desarrollaron síntomas sistémicos tuvieron un riesgo
18,7 veces mayor de presentar LCV comparado con los que no presentaron síntomas sistémicos $(\mathrm{p}<0,0001)$.

Todos los pacientes con LCV fueron hospitalizados. El 63,6\% de los pacientes con LCV evolucionó con celulitis asociada, presentando 3 veces más riesgo de desarrollar celulitis con respecto a los pacientes con LC clásico $(\mathrm{p}<0,001)$. El 8,5\% de los pacientes con LC y 9,1\% de los con LCV presentó un eritema cutáneo previo al uso de antibióticos sistémicos.

Respecto a los exámenes de laboratorio, los pacientes con LCV presentaron con mayor frecuencia leucocitosis $(\mathrm{p}=0,021)$, leucopenia $(p=0,001)$, trombocitopenia $(p<0,001)$, trombocitosis $(p=0,001)$, PCR elevada $(p=0,04)$, GPT elevada $(\mathrm{p}=0,021)$, TP disminuido $(\mathrm{p}=0,033)$, TTPA disminuido $(\mathrm{p}=0,028), \mathrm{BUN}$ elevado $(\mathrm{p}<0,001)$, creatinina plasmática elevada $(\mathrm{p}=0,006)$, hematuria $(\mathrm{p}=0,007)$, respecto a los pacientes con LC (Tabla 3). El 14\% del total de pacientes con hematuria evolucionó con LCV

Tabla 1. Características demográficas de la muestra

\begin{tabular}{|lc|}
\hline Características de la muestra & $\begin{array}{c}\text { Muestra total } \\
\mathbf{n}=\mathbf{2 0 0} \\
\mathbf{n}(\mathbf{\%})\end{array}$ \\
Edad & \\
Adultos & $187(93,5)$ \\
Niños (<15 años) & $13(6,5)$ \\
Sexo & \\
Femenino & $111(55,5)$ \\
Maculino & $89(44,5)$ \\
Tipo Loxoscelismo & \\
Cutáneo clásico & $179(89,5)$ \\
Cutáneo edematoso & $10(5)$ \\
Cutáneo visceral & $11(5,5)$ \\
Mes del año & \\
Enero & $36(18)$ \\
Febrero & $24(12)$ \\
Marzo & $20(10)$ \\
Abril & $16(8)$ \\
Mayo & $4(2)$ \\
Junio & $10(5)$ \\
Julio & $5(2,5)$ \\
Agosto & $8(4)$ \\
Septiembre & $13(6,5)$ \\
Octubre & $14(7)$ \\
Noviembre & $24(12)$ \\
Diciembre & $26(13)$ \\
\hline & \\
\hline
\end{tabular}


Caracterización 200 casos de loxoscelismo - K. Droppelmann et al

Tabla 2. Síntomas sistémicos según tipo de loxoscelismo

\begin{tabular}{|lcccc|}
\hline Síntomas sistémicos & $\begin{array}{c}\text { Loxoscelismo } \\
\text { cutáneo } \\
\mathbf{n}(\%)\end{array}$ & $\begin{array}{c}\text { Loxoscelismo } \\
\text { cutáneo-visceral } \\
\mathbf{n ~ ( \% )}\end{array}$ & Total & Valor $\mathbf{p}$ \\
\hline Fiebre & $14(7,4)$ & $8(72,7)$ & $22(11)$ & $<0,001$ \\
Ictericia & $1(0,5)$ & $1(9)$ & $2(2)$ & 0,006 \\
\hline Síntomas Gl (dolor abdominal, diarrea, vómitos) & $3(1,6)$ & $2(18)$ & $5(2,5)$ & $<0,001$ \\
\hline Coluria & $1(0,5)$ & $1(9,1)$ & $2(1)$ & 0,006 \\
\hline Algún síntoma sistémico & $17(9)$ & $8(72,7)$ & $25(12,5)$ & $<0,001$ \\
\hline
\end{tabular}

Tabla 3. Hallazgos clínicos y de laboratorio según tipo de loxoscelismo

\begin{tabular}{|c|c|c|c|c|}
\hline $\begin{array}{l}\text { Hallazgo clínico o } \\
\text { de laboratorio }\end{array}$ & $\begin{array}{c}\text { Loxoscelismo } \\
\text { cutáneo (\%) }\end{array}$ & $\begin{array}{c}\text { Loxoscelismo } \\
\text { cutáneo-visceral (\%) }\end{array}$ & $\begin{array}{l}\text { Total } \\
(\%)\end{array}$ & Valor $p$ \\
\hline Hospitalización & 16,9 & 100 & 21,5 & $<0,001$ \\
\hline Rash previo uso ATB & 8,5 & 9 & 8,5 & 0,94 \\
\hline Celulitis & 21,2 & 63,6 & 23,5 & 0,001 \\
\hline $\mathrm{Hb}$ baja & 50,9 & 36,4 & 49,6 & 0,36 \\
\hline Reticulocitos $>1,5 \%$ & 43,8 & 57,1 & 47,8 & 0,55 \\
\hline Leucocitosis & 22,7 & 54,6 & 25,6 & 0,021 \\
\hline Leucopenia & 0 & 9,1 & 0,8 & 0,001 \\
\hline Trombocitopenia & 2,7 & 27,3 & 5 & $<0,001$ \\
\hline Trombocitosis & 0 & 9,1 & 0,8 & 0,001 \\
\hline VHS elevada & 8,6 & 12,5 & 9 & 0,71 \\
\hline PCR elevada & 44,6 & 90,9 & 51,3 & 0,004 \\
\hline LDH elevada & 21,4 & 45,5 & 25,4 & 0,094 \\
\hline GOT elevada & 10,5 & 27,3 & 12,3 & 0,11 \\
\hline GPT elevada & 25 & 60 & 28,7 & 0,021 \\
\hline GGT elevada & 40,5 & 70 & 44,1 & 0,078 \\
\hline Bl total elevada & 9,7 & 30 & 11,7 & 0,057 \\
\hline BI Indirecta elevada & 9,7 & 30 & 11,7 & 0,057 \\
\hline TP bajo & 15,4 & 44,4 & 18,4 & 0,033 \\
\hline TP prolongado & 2,6 & 0 & 2,3 & 0,63 \\
\hline TTPA bajo & 30,4 & 80 & 35,3 & 0,028 \\
\hline BUN elevado & 0 & 30 & 4,6 & $<0,001$ \\
\hline Creatinina elevada & 2 & 18,2 & 3,5 & 0,006 \\
\hline$\geq 3$ GR en orina & 32,5 & 72,7 & 35,2 & 0,007 \\
\hline CK total elevada & 10 & 22,2 & 12,8 & 0,34 \\
\hline
\end{tabular}


Tabla 4. Tratamientos utilizados según tipo de loxoscelismo

\begin{tabular}{|lcccc|}
\hline Tratamientos utilizados & $\begin{array}{c}\text { Loxocelismo } \\
\text { cutáneo (\%) }\end{array}$ & $\begin{array}{c}\text { Loxocelismo } \\
\text { cutáneo-visceral (\%) }\end{array}$ & $\begin{array}{c}\text { Total } \\
\text { (\%) }\end{array}$ & Valor p \\
\hline Analgesia & 74,1 & 72,7 & 74 & 0,92 \\
\hline Antihistamínicos & 46 & 54,6 & 46,5 & 0,58 \\
\hline Corticoides tópicos & 11,6 & 36,4 & 13 & $\mathbf{0 , 0 1 8}$ \\
Corticoides sistémicos & 25,4 & 81,8 & 28,5 & $<\mathbf{0 , 0 0 1}$ \\
\hline Colchicina & 0,5 & 18,2 & 1,5 & $<0,001$ \\
\hline Dapsona & 10,6 & 27,3 & 11,5 & 0,09 \\
\hline Mupirocina & 1,6 & 0 & 1,5 & 0,67 \\
\hline Antibióticos sistémicos & 39,4 & 81,8 & 41,7 & $\mathbf{0 , 0 0 6}$ \\
\hline Vancomicina & 0,5 & 9,1 & 1 & $\mathbf{0 , 0 0 6}$ \\
\hline Ampicilina-Sulbactam & 1,1 & 9,1 & 1,5 & $\mathbf{0 , 0 3 3}$ \\
\hline Amoxicilina-Ac. clavulánico & 1,6 & 9,1 & 2 & 0,084 \\
\hline Ciprofloxacino & 2,1 & 0 & 2 & 0,63 \\
\hline Clindamicina & 4,8 & 27,3 & 6 & $\mathbf{0 , 0 0 2}$ \\
\hline Doxiciclina & 1,1 & 9,1 & 1,5 & $\mathbf{0 , 0 3 3}$ \\
\hline Cefadroxilo & 27 & 27,3 & 27 & 0,98 \\
\hline Cefazolina & 7,4 & 45,5 & 9,5 & $<\mathbf{0 , 0 0 1}$ \\
\hline Cloxacilina & 2,7 & 0 & 2,5 & 0,59 \\
\hline Ceftriaxona & 2,1 & 9,1 & 2,5 & 0,15 \\
\hline
\end{tabular}

mientras que solo $2,9 \%$ del total de pacientes que no tuvo hematuria presentó un LCV $(\mathrm{p}=0,007)$. Los pacientes con hematuria tuvieron 4,9 veces más riesgo de presentar LCV en comparación con los que no presentan hematuria, siendo esta asociación estadísticamente significativa $(\mathrm{p}=0,023)$. No todos los pacientes con hematuria evolucionan con LCV (32,5\% de los pacientes con LC tuvo hematuria).

El 74\% de los pacientes recibió analgesia oral o endovenosa, $46,5 \%$ antihistamínicos y $28,5 \%$ corticoides sistémicos. El 41,7\% fue tratado con antibióticos sistémicos, y de ellos, cefadroxilo fue el más usado en $27 \%$ de los casos. Con respecto al tratamiento, se observaron diferencias estadísticamente significativas entre pacientes con LCy LCV, $81,8 \%$ de los pacientes con LCV fue tratado con corticoides y antibióticos sistémicos $(\mathrm{p}<0,001$ y $\mathrm{p}=0,006$ respectivamente). Otros antibióticos como vancomicina, ampicilina-sulbactam, clin- damicina, doxiciclina y cefazolina fueron más frecuentemente utilizados en LCV que en LC $(\mathrm{p}<0,05)$ (Tabla 4).

En ningún paciente se utilizó suero antiloxosceles.

\section{Discusión}

Resultó relevante caracterizar los casos de loxoscelismo que consultaron a nuestro centro, ya que, además de ser una enfermedad prevalente en Chile, los únicos registros nacionales con lo que contamos son la revisión que hubo entre los años 1950 y 2000, publicada por Schenone et al (206 casos $)^{20}$, y el registro telefónico de las consultas al CITUC publicada por Ríos et al (2.831 consultas $)^{19}$. Dentro de nuestros resultados pudimos observar algunos datos similares y comparables con estos estudios, como: un amplio rango etario, proporción similar entre ambos sexos, la mayoría 
de los pacientes visualizó la araña, y una mayor prevalencia de mordeduras en las extremidades. También destacamos una tendencia estival de presentación de las mordeduras.

Si bien en Chile se ha descrito una letalidad de $1-3 \%$ para LC y de $20-25 \%$ para $L_{C V}{ }^{20}$, en nuestro estudio no se registraron muertes por loxoscelismo. Esto pudiera deberse, en parte, a que la población consultó en forma temprana (la gran mayoría dentro de las primeras $24 \mathrm{~h}$ ), y por la disponiblidad de una guía MINSAL para el reconocimiento precoz y el tratamiento del $\mathrm{LCV}^{22}$. Otra causa posible es que tuvimos una menor prevalencia de casos de LCV (5,5\% del total de casos), respecto a lo descrito en la literatura $(18 \%$ del total de casos) $)^{20}$. Esta menor proporción de LCV pudiera deberse a mayor consulta de la población debido a todas las formas clínicas de loxoscelismo. Puede que en los años en que fueron realizados los estudios anteriores, haya habido una mayor proporción LC que nunca consultó.

Existen interrogantes relevantes que se podrían resolver en estudios futuros. La primera es definir con mayor claridad el diagnóstico de LC. Si bien es aceptado que el diagnóstico es clínico, basado en la presentación epidemiológica, síntomas, lesión cutánea característica y evolución, falta consenso para tener criterios diagnósticos que ayuden tanto al reconocimiento como a la futura investigación de la enfermedad. Recientemente se publicó la mnemotecnia NOT RECLUSE para el descarte del diagnóstico, pero su uso como test diagnóstico no ha sido validado aún ${ }^{8}$. Tampoco existen criterios diagnósticos establecidos en el LCV.

Dentro de los exámenes de laboratorio hubo mayor frecuencia de leucocitosis, leucopenia, trombocitopenia, trombocitosis, elevación de la PCR, elevación de la GPT, disminución en el TP y TTPA, elevación del BUN y creatinina plasmática, y presencia de hematuria en los pacientes con LCV respecto a los pacientes con LC. Por otro lado, encontramos que $72,7 \%$ de los pacientes con LCV tuvo síntomas sistémicos (valor $\mathrm{p}<0,001$ ). Esto nos hace concluir dos cosas: faltan criterios diagnósticos para predecir/establecer el diagnóstico de LCV y estos criterios podrían basarse en la presencia de síntomas sistémicos y hallazgos de laboratorio, buscando la presencia de inflamación, signos indirectos de hemólisis y de falla renal aguda. La presencia de síntomas sistémicos asociados es un dato relevante para identificar a los pacien- tes más graves. Las guías MINSAL actuales solo nombran los síntomas sistémicos y recomiendan hospitalizar en una unidad de tratamiento intensivo a todo paciente con hemoglobinuria, dándole un alto valor al examen de orina ${ }^{22}$.

Los pacientes que presentaron hematuria tuvieron 4,9 veces más riesgo de presentar $\mathrm{LCV}$ en comparación con los pacientes que no tuvieron hematuria, y los pacientes con síntomas sistémicos tuvieron 18,7 veces más riesgo de presentar LCV en comparación con los pacientes sin síntomas sistémicos. Sin embargo, no todos los pacientes con hematuria evolucionaron a un LCV $(32,5 \%$ de los LC tuvo hematuria). El 27,3\% de los pacientes hospitalizados con LCV nunca presentó hematuria y sí otras alteraciones de laboratorio indicadoras de hemólisis, inflamación y falla renal aguda. Por lo tanto, proponemos que para reconocer a un paciente con LCV, inicialmente se deberían buscar síntomas sistémicos como fiebre, ictericia, dolor abdominal, vómitos, coluria. Luego, en los exámenes de laboratorio, se deberían buscar signos de hemólisis, falla renal aguda, inflamación y hematuria. Sugerimos, que un paciente con síntomas sistémicos y sin hematuria debería ser vigilado, porque la ausencia de hematuria no descarta la evolución a un LCV. Por otro lado, si consideramos que $32,5 \%$ de los pacientes con LC tuvo hematuria, es difícil considerarla como único factor en la decisión de hospitalización en una unidad de alta complejidad en el contexto de un loxoscelismo. Estas incógnitas pueden complejizar las decisiones terapéuticas para los médicos de urgencia y antención primaria. Estudios prospectivos podrían permitir evaluar cuál de estas variables logra predecir mejor la evolución a un LCV.

Con respecto al tratamiento, encontramos que la analgesia fue requerida en $74 \%$ de los pacientes. Habitualmente no se discute en las guías clínicas y es de suma relevancia el manejo del dolor en estos pacientes. Los corticoides y antibióticos fueron ampliamente indicados en pacientes con LCV. En ningún paciente se usó el suero antiloxosceles. Ya en el año 1988, Schenone et al. desincentivaban su uso por futilidad. Los pacientes generalmente consultan fuera de la ventana terapéutica para el uso de suero antiloxosceles, y en las guías MINSAL del año 2016 ya no se recomienda su uso ${ }^{22}$.

Encontramos 63,6\% de desarrollo de celulitis asociada en el LCV, lo que apoyaría el uso precoz de antibióticos en pacientes que se van a hospitalizar. 
Como este estudio es retrospectivo, no nos permite cambiar conductas en el manejo ni definición de un loxoscelismo, pero al caracterizar las variables estudiadas, permite identificar los principales problemas que se debieran tener en cuenta al enfrentarse a un caso de loxoscelismo.

A pesar de que el loxoscelismo es un problema importante de salud en nuestro país, aún quedan muchas interrogantes por responder, principalmente en el diagnóstico y tratamiento de la enfermedad. Se requieren estudios futuros que validen criterios diagnósticos para LC y LCV, estudios que evalúen cuáles son las mejores variables para predecir un LCV, y estudios que evalúen la respuesta a tratamientos en pacientes con LC y LCV.

\section{Referencias}

1. Isbister GK, Fan HW. Spider bite. Lancet 2011; 378 (9808): 2039-47.

2. Schenone H. Cuadros tóxicos producidos por mordeduras de araña en Chile: latrodectismo y loxoscelismo. Rev Med Chile 2003; 131: 437-44.

3. Cabrerizo S, Docampo PC, Cari C, et al. Loxoscelismo: epidemiología y clínica de una patología endémica en el país. Arch Argent Pediatr 2009; 107 (2): 152-9.

4. Zimmerman SP, Rothman JA, Hansen JL, Rusin MM, Bertone MA, Hamrick HJ. Systemic loxoscelism in a nonendemic area: a diagnostic challenge for the unsuspecting physician. Clin Pediatr (Phila) 2014; 53 (11): 1098-100.

5. Swanson DL, Vetter RS. Bites of brown recluse spiders and suspected necrotic arachnidism. N Engl J Med 2005; 352 (7): 700-7.

6. Andersen RJ, Campoli J, Johar SK, Schumacher KA, Allison EJ Jr. Suspected brown recluse envenomation: a case report and review of different treatment modalities. J Emerg Med 2011; 41 (2): e31-7.

7. Saucier JR. Arachnid envenomation. Emerg Med Clin North Am 2004; 22 (2): 405-22.

8. Stoecker WV, Vetter RS, Dyer JA. NOT RECLUSE-A Mnemonic Device to Avoid False Diagnoses of Brown Recluse Spider Bites. Jama Dermatol 2017; 153 (5): 3778.

9. Swanson DL, Vetter RS. Loxoscelism. Clin Dermatol 2006; 24 (3): 213-21.

10. Gertsch W, Ennik F. The spider genus Loxosceles in North America, Central America and the West Indies (Araneae Loxoscelidae). Bull A Mus Nat His 1983; 175: 264-360.
11. Nentwig W, Pantini P, Vetter RS. Distribution and medical aspects of Loxosceles rufescens, one of the most invasive spiders of the world (Araneae: Sicariidae). Toxicon 2017; 132: 19-28.

12. Southcott RV. Spiders of the genus Loxosceles in Australia. Med J Aust 1976; 1 (12): 406-8.

13. Futrell JM. Loxoscelism. Am J Med Sci 1992; 304 (4): 261-7.

14. Manríquez JJ, Silva S. Loxoscelismo cutáneo y cutáneo-visceral: Revisión sistemática. Rev Chilena Infectol 2009; 26 (5): 420-32.

15. Zambrano A, González J, Callejas G. Desenlace fatal por loxoscelismo cutáneo visceral. Rev Med Chile 2005; 133 (2): 219-223.

16. Harz-Fresno I, Manterola P, Ru Z M, Abud C. Loxoscelismo cutáneo visceral: actualización en el manejo a propósito de un caso. Rev Chilena Infectol 2015; 32 (2): 230-3.

17. Machiavello A. La Loxosceles laeta, causa del aracnoidismo cutáneo o mancha grangrenosa de Chile. Rev Ch Hist Nat 1937; 41: 11-9.

18. Macchiavello A. Cutaneous arachnidism or gangrenous spot of Chile. Puerto Rico J Pub Health 1947; 22:425.

19. Ríos JC, Pérez M, Sánchez P, Bettini M, Mieres JJ, Paris E. Caracterización clínico-epidemiológica telefónica de la mordedura por araña de rincón, en un centro de información toxicológica de Chile, durante el año 2005. Rev Med Chile 2007; 135 (9): 1160-5.

20. Schenone H, Saavedra T, Rojas A, Villarroel F. Loxoscelismo en Chile. Estudios epidemiológicos, clínicos y experimentales. Rev Inst Med Trop Sao Paulo 1989; 31 (6): 403-15.

21. Rees R, Campbell D, Rieger E, King LE. The diagnosis and treatment of brown recluse spider bites. Ann Emerg Med 1987: 16 (9): 945-9.

22. Guía para el Manejo de Mordedura de Araña de los Rincones - Loxosceles laeta. Chile, 2016.

23. Canals M, Taucare-Rios A, Brescovit AD, et al. Niche modelling of the Chilean recluse spider Loxosceles laeta and araneophagic spitting spider Scytodes globula and risk for loxoscelism in Chile. Med Vet Entomol 2016; 30 (4): 383-91.

24. Schenone H. Loxoscelismo cutáneo de predominio edematoso. Bol Chil Parasitol 1998; 53 (3-4): 78-83.

25. De la Barra P, Vial V, Labraña Y, Álvarez AM, Seguel H. Loxoscelismo cutáneo predominantemente edematoso: a propósito de un caso. Rev Chilena Infectol 2015; 32 (4): 467-71.

26. Canals M, Solís R. Desarrollo de cohortes y parámetros poblacionales de la araña del rincón Loxosceles laeta. Rev Chilena Infectol 2014; 31(5): 555-62. 